\title{
Convex Independence and the Structure of Clone-Free Multipartite Tournaments
}

\author{
Darren B. Parker* Randy F. Westhoff ${ }^{\dagger} \quad$ Marty J. Wolf ${ }^{\ddagger}$ \\ MR Subject Classifications: 05C20, 06B99 \\ Keywords: Convex sets, rank, Helly number, Radon number, multipartite tournaments
}

\begin{abstract}
We investigate the convex invariants associated with two-path convexity in clonefree multipartite tournaments. Specifically, we explore the relationship between the Helly number, Radon number and rank of such digraphs. The main result is a structural theorem that describes the arc relationships among certain vertices associated with vertices of a given convexly independent set. We use this to prove that the Helly number, Radon number, and rank coincide in any clone-free bipartite tournament. We then study the relationship between Helly independence and Radon independence in clone-free multipartite tournaments. We show that if the rank is at least 4 or the Helly number is at least 3, then the Helly number and the Radon number are equal.
\end{abstract}

\section{Introduction}

Several notions of convexity in graphs and digraphs have been investigated. In each case, the convex sets are defined in terms of a particular type of path. Let $T=(V, E)$ be a graph or digraph and let $\mathcal{P}$ be a set of paths in $T$. A subset $A \subseteq V$ is $\mathcal{P}$-convex if, whenever $v, w \in A$, any path in $\mathcal{P}$ that originates at $v$ and ends at $w$ can involve only vertices in $A$. The most commonly studied type of convexity is geodesic convexity where $\mathcal{P}$ is taken to be the set of geodesics in $T$ (see [CZ99], [CFZ02], [ES85] and [HN81]). Other types of convexity that have been studied include induced path convexity where $\mathcal{P}$ is the set of all chordless paths (see [Duc88]), path convexity (see [Nie81] and [Pfa71]) and triangle path convexity (see [CM99]). In this paper we will consider two-path convexity

*Department of Mathematics, Grand Valley State University, Allendale, MI 49401-6495, parkerda@gvsu.edu, http://faculty.gvsu.edu/parkerda/

$\dagger$ 'Department of Mathematics \& Computer Science, Bemidji State University, Bemidji, MN 56601, rwesthoff@bemidjistate.edu

${ }_{\ddagger}^{\ddagger}$ Department of Mathematics \& Computer Science, Bemidji State University, Bemidji, MN 56601, mjwolf@bemidjistate.edu, http//cs.bemidjistate.edu/mjwolf 
where $\mathcal{P}$ is taken to be the set of all 2-paths in a digraph $T$. Two-path convexity was first studied in tournaments in [EFHM72], [Var76] and [Moo72] and more recently in multipartite tournaments in [PWWb], [PWWd], [ADEP05], and [PWWc].

More generally, a convexity space is a pair $\mathrm{C}=(V, C)$, where $V$ is a set and $C$ is a collection of subsets of $V$ such that $\emptyset, V \in C$ and $C$ is closed under arbitrary intersections and nested unions. Note that the vertex set of a graph or digraph along with the set of all $\mathcal{P}$-convex subsets forms a convexity space for any set of paths $\mathcal{P}$. For a subset $S \subseteq V$, the convex hull of $\mathrm{S}$, denoted $C(S)$, is defined to be the smallest convex subset containing $S$.

Several numerical invariants can be associated to a convexity space $\mathcal{C}=(V, C)$. Three of the most studied are the Helly, Radon and Caratheodory numbers (see [JN84], [Pol95], and [CM99]). These can each be defined using notions of independence (see [vdV93, Chap. 3]). Let $F \subseteq V$. We say $F$ is $H$-independent if $\bigcap_{p \in F} C(F-\{p\})=\emptyset$. The Helly number $h(\mathcal{C})$ is the size of a largest $H$-independent set. A partition $F=A \cup B$ with $C(A) \cap C(B) \neq \emptyset$ is called a Radon partition of $F$, and $F$ is $R$-independent if $F$ does not have a Radon partition. The Radon number $r(\mathcal{C})$ is the size of a largest $R$-independent set. Note that some authors define the Radon number to be the smallest number $r$ such that every subset of size $r$ has a Radon partition. This results in a Radon number one larger than ours. It is well-known that if $F$ is $H$-independent then $F$ is $R$-independent. (see [vdV93, p. 163]). This implies Levi's inequality, which is $h(\mathcal{C}) \leq r(\mathcal{C})$.

A set $F$ is $C$-independent if $C(F) \nsubseteq \cup_{a \in F} C(F-\{a\})$ and the Caratheodory number $c(\mathcal{C})$ is the size of the largest $C$-independent set. Equivalently, the Caratheodory number can be defined as the smallest number $c$ such that for every $S \subseteq V$ and $p \in C(S)$, there is an $F \subseteq S$ with $|F| \leq c$ such that $p \in C(F)$. One final type of independence we will consider is convex independence. $F$ is convexly independent if, for each $p \in F$, we have $p \notin C(F-\{p\})$. The rank $d(\mathcal{C})$ is the size of a largest convexly independent set. Rank provides an upper bound on the number of elements of a convex set which are needed to generate the convex set using convex hulls. In [HW96], D. Haglin and M. Wolf used the fact that the collection of two-path convex subsets in a tournament has rank 2 to construct an algorithm for computing the convex subsets of a given tournament. Note that since any set that is $H$-, $R$ - or $C$-independent must also be convexly independent, rank is an upper bound for the Helly, Radon and Caratheodory numbers. In particular, with Levi's inequality we have that $h(\mathcal{C}) \leq r(\mathcal{C}) \leq d(\mathcal{C})$.

Let $T=(V, E)$ be a digraph with vertex set $V$ and arc set $E$. We denote an arc $(v, w) \in E$ by $v \rightarrow w$ and say that $v$ dominates $w$. If $U, W \subseteq V$, then we write $U \rightarrow W$ to indicate that every vertex in $U$ dominates every vertex in $W$. We call $T$ a multipartite tournament if it is possible to partition $V$ into partite sets $P_{1}, P_{2}, \ldots, P_{k}, k \geq 2$ such that there is precisely one arc between each pair of vertices in different partite sets and no arcs between vertices in the same partite set. In the case when $k=2$ we will also call $T$ a bipartite tournament. Two vertices are clones if they have identical insets and outsets, and $T$ is clone-free if it has no clones. In a multipartite tournament, this is equivalent to every pair of vertices in the same partite set being distinguished by another vertex (i.e. having a two-path between them). If $A, B \in \mathcal{C}(T)$, we denote the convex hull of $A \cup B$ 
by $A \vee B$. If $v, w \in V$, we drop the set notation and write $\{v\} \vee\{w\}$ as $v \vee w$. Finally, we denote by $T^{*}$ the digraph with the same vertex set as $T$, and where $(v, w)$ is an arc of $T^{*}$ if and only if $(w, v)$ is an $\operatorname{arc}$ of $T$.

\section{Convexly Independent Sets in Multipartite Tour- naments}

In [PWWb], we studied the properties of convexly independent sets under two-path convexity in multipartite tournaments. In this section, we present results from that paper that will be important in our study of clone-free multipartite tournaments.

Let $T=(V, E)$ be a clone-free multipartite tournament, and let $U \subseteq V$ be a convexly independent set. We showed in [PWWb, Lem. 3.1(2)] that $U$ can have a nonempty intersection with at most two partite sets. Thus $T$ has partite sets $P_{0}$ and $P_{1}$ such that $A=U \cap P_{0}$ and $B=U \cap P_{1}$ with $U=A \cup B$. By [PWWb, Lem. 3.1(1)], we must have $A \rightarrow B$ or $B \rightarrow A$. Note that $T$ and $T^{*}$ have the same convex subsets, so by relabelling $P_{0}$ and $P_{1}$ and reversing the arcs, if necessary, we can assume that $|A| \geq|B|$ and $A \rightarrow B$ if $B \neq \emptyset$.

The following sets of distinguishing vertices are important to us. Let $C \subseteq V$. We define

$$
\begin{aligned}
& D_{C}^{\vec{C}}=\{z \in V: z \rightarrow x \text { for some } x \in C, y \rightarrow z \text { for all } y \in C-\{x\}\} \\
& D_{C}^{\leftarrow}=\{z \in V: z \leftarrow x \text { for some } x \in C, z \rightarrow y \text { for all } y \in C-\{x\}\}
\end{aligned}
$$

The following appears in [PWWd].

Theorem 2.1. Let $T$ be a clone-free multipartite tournament. Let $A$ and $B$ form a convexly independent set, with $A \rightarrow B$ when both sets are nonempty.

1. If $A=\left\{x_{1}, \cdots, x_{m}\right\}, m \geq 2$, then one can order the vertices in $A$ such that there exist $u_{2}, \cdots, u_{m} \in D_{\vec{A}}$ (resp., in $D_{A}^{\leftarrow}$ if $D_{A}=\emptyset$ ) such that $u_{i} \rightarrow x_{i}$ (resp., $x_{i} \rightarrow u_{i}$ ).

2. If $|A| \geq 3$, then $D_{A} \overrightarrow{\neq} \neq \emptyset$ if and only if $D_{A}^{\leftarrow}=\emptyset$, and $D_{\vec{A}}$ and $D_{A}^{\leftarrow}$ each lie in at most one partite set.

3. Suppose $A, B \neq \emptyset$. If $|A| \geq 2$, then $D_{A}$ is in the same partite set as $B$, and if $|B| \geq 2$, then $D_{B}^{\leftarrow}$ is in the same partite set as $A$.

4. If $|A|,|B| \geq 2$, then $D_{B}^{\leftarrow} \rightarrow D_{A}$.

5. Any vertex that distinguishes vertices in $A$ must be in either $D_{A}^{\rightarrow}$ or $D_{A}^{\leftarrow}$ and any vertex that distinguishes vertices in $B$ must be in $D_{B}^{\leftarrow}$ or $D_{B}$. If $A, B \neq \emptyset$, then any vertex that distinguishes vertices in $A$ must be in $D_{A}$ and any vertex that distinguishes vertices in $B$ must be in $D_{B}^{\leftarrow}$. 


\section{Structure of Clone-Free Multipartite Tournaments}

Let $T=(V, E)$ be a clone-free multipartite tournament and let $U$ be a convexly independent subset of $T$. As before. let $P_{0}$ and $P_{1}$ be partite sets of $T, A=U \cap P_{0}$ and $B=U \cap P_{1}$ such that $U=A \cup B$. We may assume that $|A| \geq|B|$ and $A \rightarrow B$ when $B \neq \emptyset$. By Theorem 2.1(2), one of $D_{A}$ or $D_{A}^{\leftarrow}$ is nonempty, and when $|A| \geq 3$, the other is empty. In the case of $B=\emptyset$, we choose $T$ or $T^{*}$ such that $D_{A} \neq \emptyset$ and let $P_{1}$ be the partite set containing $D_{A}$. We will assume these notational conventions and choices have been made throughout the remainder of the paper.

Ordinarily, the convex hull of a set $U \subseteq V$ is constructed using the sets $C_{k}(U)$, defined as follows. Let $C_{0}(U)=U$ and for $k \geq 1$, let

$$
C_{k}(U)=C_{k-1}(U) \cup\left\{w \in V: x \rightarrow w \rightarrow y \text { for some } x, y \in C_{k-1}(U)\right\}
$$

Then $C(U)=\bigcup_{k=0}^{\infty} C_{k}(U)$. We will construct the convex hull somewhat differently here. Define $\Delta_{k}(U)$ as follows. Let $\Delta_{0}(U)=A, \Delta_{1}(U)=B \cup C_{1}(A)$, and for $t \geq 2$, let $\Delta_{t}(U)=C_{1}\left(\Delta_{t-1}(U)\right)$. Note that $C(U)=\bigcup_{i=0}^{\infty} \Delta_{i}(U)$.

Our goal is to create pairwise disjoint subsets of $C(U)$, each of which is associated with a given $x \in U$. We do this as follows.

Definition 3.1. Let $U=A \cup B$ be a convexly independent set with $A \rightarrow B$. For each $x \in U$, define $\bar{D}_{t}(x)$ for $t \geq 0$ as follows. If $x \in A$, then $\bar{D}_{0}(x)=\{x\}$, and if $x \in B$, then $\bar{D}_{0}(x)=\emptyset$ and $\bar{D}_{1}(x)=\{x\}$. Otherwise, we have

$$
\begin{aligned}
\bar{D}_{2 k}(x) & =\left\{v \in \Delta_{2 k}(U): u \rightarrow v \text { for some } u \in \bar{D}_{2 \ell-1}(x), \ell \leq k\right\} \\
\bar{D}_{2 k+1}(x) & =\left\{v \in \Delta_{2 k+1}(U): v \rightarrow u \text { for some } u \in \bar{D}_{2 \ell}(x), \ell \leq k\right\}
\end{aligned}
$$

We then define $D_{t}(x)=\bigcup_{k \leq t} \bar{D}_{k}(x)$ and $D(x)=\bigcup_{t=0}^{\infty} D_{t}(x)$.

Notice that $\bar{D}_{k}(x) \subseteq \bar{D}_{k+2}(x)$ for $k \geq 1$ if $x \in A$, and for $k \geq 2$ if $x \in B$. The following lemma relates the notation introduced above to the notation used in Section 2.

Lemma 3.2. Let $U=A \cup B$ be a convexly independent set. Then $D_{A}=\bigcup_{x \in A} \bar{D}_{1}(x)$ and $D_{B}^{\leftarrow}=\bigcup_{x \in B} \bar{D}_{2}(x)$

Proof. Clearly, $D_{A} \subseteq \bigcup_{x \in A} \bar{D}_{1}(x)$. Let $u \in \bar{D}_{1}(x)$ for some $x \in A$. Then $u \in C_{1}(A)$ and $u \rightarrow x$. By Theorem 2.1(5), $u \in D_{A}$. To show that $D_{B}^{\leftarrow}=\bigcup_{x \in B} \bar{D}_{2}(x)$, note that $D_{B}^{\leftarrow} \subseteq \bigcup_{x \in B} \bar{D}_{2}(x)$ and if $u \in \bar{D}_{2}(x)$ for some $x \in B$ then $u \in C_{1}\left(B \cup C_{1}(A)\right)$ and $x \rightarrow u$. If $u \in C_{1}(B)$ then $u \in D_{B}^{\leftarrow}$ by Theorem 2.1(5) and if $|B|=1$ then by default $u \in D_{B}^{\leftarrow}$. If not, then $|B| \geq 2, B \rightarrow u$ and there is a $y \in C_{1}(A)$ such that $u \rightarrow y$. Since $x \rightarrow u \rightarrow y$ and $A \rightarrow B \rightarrow u$ then $B \subseteq C(A \cup\{x\})$ which is a contradiction.

Given a convexly independent set $U$ with $|U| \geq 3$, the next two lemmas allow us to replace vertices in $U \cap P_{0}$ with related vertices in $P_{1}$ or vertices in $U \cap P_{1}$ with related vertices in $P_{0}$ without changing the structure of the $D(x)$ 's. 
Lemma 3.3. Let $U=A \cup B$ be a convexly independent set with $|A| \geq 3$. Let $x \in U$, $w \in \bar{D}_{i}(x)$, where $i=1$ if $x \in A$ and $i=2$ if $x \in B$. Let $W=(U-\{x\}) \cup\{w\}$.

1. If $x \in A$, then $\Delta_{k}(W) \subseteq \Delta_{k}(U) \subseteq \Delta_{k+2}(W)$ for all $k \geq 0$.

2. If $x \in B$, then $\Delta_{k}(U) \subseteq \Delta_{k}(W) \subseteq \Delta_{k+2}(U)$ for all $k \geq 0$.

Proof. For (1), first note that $\Delta_{0}(W)=A-\{x\} \subseteq A=\Delta_{0}(U)$. Since $|A| \geq 3$, Theorem 2.1(1) implies $D_{\overrightarrow{A-\{x\}}}^{\vec{x}} \neq \emptyset$. Then $w \rightarrow x \rightarrow D_{A-\{x\}}$ gives us $x \in C_{1}\left(D_{\vec{A}-\{x\}} \cup\{w\}\right) \subseteq$ $\Delta_{2}(W)$, which proves the case $k=0$. Since $w \in \Delta_{1}(U), \Delta_{k}(W) \subseteq \Delta_{k}(U)$ for any $k \geq 1$. Moreover, $x \in \Delta_{2}(W)$ implies $C_{1}(U) \subseteq \Delta_{3}(U)$, and thus $\Delta_{1}(U) \subseteq \Delta_{3}(W)$. The fact that $\Delta_{k}(U) \subseteq \Delta_{k+2}(W)$ for $k \geq 2$ follows easily by induction. The proof of part (2) is similar.

Lemma 3.4. Let $U=A \cup B$ be a convexly independent set with $|A| \geq 3$. Let $x \in U$, $w \in \bar{D}_{i}(x)$, where $i=1$ if $x \in A$ and $i=2$ if $x \in B$.

1. $(U-\{x\}) \cup\{w\}$ is convexly independent.

2. If we replace $U$ with $W=(U-\{x\}) \cup\{w\}$ and let $\bar{D}_{t}^{\prime}(y), D^{\prime}(y)$ be the analogous sets for $y \in W$, then $\cup_{k=0}^{\infty} D_{2 k}(y)=\cup_{k=0}^{\infty} D_{2 k}^{\prime}(y)$ and $\cup_{k=0}^{\infty} D_{2 k+1}(y)=\cup_{k=0}^{\infty} D_{2 k+1}^{\prime}(y)$ for $y \in W-\{w\}$ and $\cup_{k=0}^{\infty} D_{2 k}(x)=\cup_{k=0}^{\infty} D_{2 k}^{\prime}(w)$ and $\cup_{k=0}^{\infty} D_{2 k+1}(x)=\cup_{k=0}^{\infty} D_{2 k+1}^{\prime}(w)$.

Proof. For (1), we need only show that $w \notin C(U-\{x\})$ and that, for all $y \in U-\{x\}$, $y \notin C([U \cup\{w\}]-\{x, y\})$.

We first consider the case $x \in A$. Then there exists some $z_{1} \in A-\{x\}$. Note that $z_{1} \rightarrow w \rightarrow x$. For contradiction, suppose $w \in C(U-\{x\})$. Since $|A| \geq 3$ there is a $z_{2} \in A-\left\{x, z_{1}\right\}$. By Theorem 2.1(1), at least one of $\bar{D}_{1}\left(z_{1}\right)$ or $\bar{D}_{1}\left(z_{2}\right)$ is nonempty. Without loss of generality, suppose $v \in \bar{D}_{1}\left(z_{1}\right)$, so $\left\{x, z_{2}\right\} \rightarrow v \rightarrow z_{1}$. Then $z_{2} \rightarrow v \rightarrow z_{1}$ and $w \rightarrow x \rightarrow v$ imply $x \in C(U-\{x\})$, a contradiction.

Now let $y \in U-\{x\}$, and suppose that $y \in C([U \cup\{w\}]-\{x, y\})=C([U-\{x, y\}] \cup$ $\{w\})$. If we can show that $C([U-\{x, y\}] \cup\{w\}) \subseteq C(U-\{y\})$, then we get $y \in C(U-\{y\})$, a contradiction. Since $U-\{x, y\} \subseteq U-\{y\} \subseteq C(U-\{y\})$, we need only show that $w \in C(U-\{y\})$. Let $z \in A-\{x, y\}$. We have $z \rightarrow w \rightarrow x$. Since $x, z \in U-\{y\}$, we have $w \in C(U-\{y\})$, which gives us our contradiction.

In the case $x \in B$, we have $|U| \geq 4$ and $x \rightarrow w$. As before, assume $w \in C(U-\{x\})$. Let $z \in A$. Then $z \rightarrow x$, so we have $z \rightarrow x \rightarrow w$. Since $z, w \in C(U-\{x\})$, we get $x \in C(U-\{x\})$, a contradiction.

Now suppose that $y \in C([U \cup\{w\}]-\{x, y\})$ for some $y \in U-\{x\}$. As before, we need only show that this implies $w \in C(U-\{y\})$. Since $|A-\{y\}| \geq 2$, we have $D_{A-\{y\}} \neq \emptyset$. Since $x \rightarrow w \rightarrow D_{A-\{y\}}$, we have $w \in C(U-\{y\})$, and we are done. This completes the proof of (1).

For (2), it suffices to show that $\bar{D}_{k}(x) \subseteq \bar{D}_{k+2}^{\prime}(w)$ and $\bar{D}_{k}^{\prime}(w) \subseteq \bar{D}_{k+2}(x)$ for all $k \geq 0$ and that $\bar{D}_{k}(y) \subseteq \bar{D}_{k+2}^{\prime}(y)$ and $\bar{D}_{k}^{\prime}(y) \subseteq \bar{D}_{k+2}(y)$ for all $k \geq 0, y \in U-\{x\}$. We use Lemma 3.3 to prove $\bar{D}_{k}(x) \subseteq \bar{D}_{k+2}^{\prime}(u)$. The case $k=0$ is then trivial, as is $k=1$ when 
$x \in B$. For $k \geq 1$, let $v \in \bar{D}_{k}(x)$. There exists $v^{\prime} \in \bar{D}_{k-1}(x)$ with $v \rightarrow v^{\prime}$ if $k$ is odd and $v^{\prime} \rightarrow v$ if $k$ is even. By induction, $v^{\prime} \in \bar{D}_{k+1}^{\prime}(w)$. It follows that $v \in \bar{D}_{k+2}^{\prime}(w)$. The remainder of the proof follows similarly.

The following technical lemma is helpful in the proof of the main theorem.

Lemma 3.5. Let $U=A \cup B$ be a convexly independent set with $U^{\prime} \subseteq U$ and let $v \in C\left(U^{\prime}\right)$.

1. If $A \neq \emptyset$ and $v \rightarrow A$ and either $\left|U^{\prime} \cap A\right| \geq 2$ or $U^{\prime} \cap B \neq \emptyset$, then $A \subseteq C\left(U^{\prime}\right)$.

2. If $B \neq \emptyset$ and $B \rightarrow v$ and either $\left|U^{\prime} \cap B\right| \geq 2$ or $U^{\prime} \cap A \neq \emptyset$, then $B \subseteq C\left(U^{\prime}\right)$.

3. Suppose $A \rightarrow v, U^{\prime} \cap A \neq \emptyset$, and $q \in \bar{D}_{1}(z)$ for $z \in A-U^{\prime}$. Then $q \nrightarrow v$.

4. Suppose $v \rightarrow B, U^{\prime} \cap B \neq \emptyset$, and $q \in \bar{D}_{2}(z)$ for $z \in B-U^{\prime}$. Then $v \nrightarrow q$.

Proof. We begin with (1). In the case $\left|U^{\prime} \cap A\right| \geq 2$, let $x, y \in U^{\prime} \cap A$. By Lemma 2.1(1), we can assume $\bar{D}_{1}(y) \neq \emptyset$. Let $q \in \bar{D}_{1}(y) \subseteq C\left(U^{\prime}\right)$. If $z \in A-\{x, y\}$, then $v \rightarrow z \rightarrow q$, and so $z \in C\left(U^{\prime}\right)$. Thus, $A \subseteq C\left(U^{\prime}\right)$.

In the case $U^{\prime} \cap B \neq \emptyset$, let $x \in U^{\prime} \cap B$. If $y \in A$, then $v \rightarrow y \rightarrow x$, and so $y \in C\left(U^{\prime}\right)$, implying $A \subseteq C\left(U^{\prime}\right)$. Part (2) follows similarly.

For (3), let $x \in U^{\prime} \cap A$. If $q \rightarrow v$, then we have $x \rightarrow q \rightarrow v$ and $q \rightarrow z \rightarrow v$, which implies $z \in C\left(U^{\prime}\right)$, a contradiction. Part (4) follows similarly.

We can now prove our main result, which shows that that the $D(x)$ 's are contained in exactly two partite sets. Furthermore, for each $x \in U$, the vertices in $D(x)$ behave similarly to $x$ when used in the construction of convex hulls.

Theorem 3.6. Let $T$ be a clone-free multipartite tournament, and let $U=A \cup B$ be convexly independent. Suppose $|U| \geq 4$, and let $x, y, z \in U$.

1. For all $k, \ell \geq 0, \bar{D}_{2 k}(x) \subseteq P_{0}$ and $\bar{D}_{2 \ell+1}(x) \subseteq P_{1}$.

2. If $x \neq y$, then $\bar{D}_{2 k}(x) \rightarrow \bar{D}_{2 \ell+1}(y)$ for all $k, \ell \geq 0$.

3. Let $u \in \bar{D}_{r}(x), v \in \bar{D}_{s}(y)$, where $x \neq y, r$ and $s$ have the same parity. If $x, y \in A$ and $\bar{D}_{1}(x), \bar{D}_{1}(y) \neq \emptyset$ or if $x, y \in B$ and $\bar{D}_{2}(x), \bar{D}_{2}(y) \neq \emptyset$, then $x \vee y=u \vee v$.

4. Let $u \in \bar{D}_{m}(x), v \in \bar{D}_{n}(y)$, and $w \in \bar{D}_{p}(z)$, where $x, y$, and $z$ are distinct. Then $x \vee y \vee z=u \vee v \vee w$.

Proof. If $|B|=0$ or 1 , we can use Lemma 3.4 to convert $A$ and $B$ into $A^{\prime}$ and $B^{\prime}$ with $\left|A^{\prime}\right|,\left|B^{\prime}\right| \geq 2, A^{\prime} \rightarrow B^{\prime}$, and where $\cup_{k=0}^{\infty} D_{2 k}(x)$ and $\cup_{l=0}^{\infty} D_{2 l+1}(x)$ for $x \in A^{\prime} \cup B^{\prime}$ are identical to those of $U$. Thus, we can assume that $|A|,|B| \geq 2$.

We prove all statements simultaneously by induction on $\gamma=\max \{2 k, 2 \ell+1, r, s, m, n, p\}$. The results for $\gamma=0,1$ follow from the definitions and Theorem 2.1(3) and (4). Theorem 2.1 also covers every situation where $\bar{D}_{2}(\alpha)$ is in the hypothesis and $\alpha \in B$. For the remaining cases, we begin with a lemma. 
Lemma 3.7. Suppose $0 \leq t<\gamma$ and $w \in \Delta_{t}(U)$.

1. There exists $y \in U$ such that for any distinct $x, z \in U-\{y\}$, we have $w \in x \vee y \vee z$.

2. If $w \in D_{t}(u)$ for some $u \in U$, then the conclusion of (1) holds when $y=u$.

3. If $w \in D_{t}(u)$ for some $u \in U$, then for any $z \in U-\{u\}$ with $D_{t}(z) \neq\{z\}$ and $z$ in the same partite set as $u$, we have $w \in u \vee z$.

4. If $x \in A$ (resp. $x \in B$ ) and $D_{t}(x) \neq\{x\}$ for some $t$, then $\bar{D}_{1}(x) \neq \emptyset$ (resp. $\left.\bar{D}_{2}(x) \neq \emptyset\right)$.

Proof. First note that, by induction, we can assume all the conclusions of Theorem 3.6. We first prove (2) and (3). Let $w \in D_{t}(u)$ for some $u \in U$. Then $w \in \bar{D}_{s}(u)$ for some $s \leq t$. The results are trivial if $w=u$ so assume $w \neq u$ and thus $s, t \geq 1$. For (2), we can use Theorem 3.6(4) to get $w \in x \vee w \vee z=x \vee u \vee z$. For (3), let $z \in U-\{u\}$ with $D_{t}(z) \neq\{z\}$ and $z$ in the same partite set as $u$. Assume, without loss of generality, that $u, z \in A$. If $s$ is even, Theorem 3.6(3) gives us $w \in w \vee z=u \vee z$. If $s$ is odd, let $w^{\prime} \in \bar{D}_{s-1}(u)$ with $w \rightarrow w^{\prime}$. Then $z \rightarrow w$ by Theorem 3.6(2) and $w^{\prime} \in w^{\prime} \vee z=u \vee z$ by Theorem 3.6(3). Thus, $z \rightarrow w \rightarrow w^{\prime}$ implies $w \in w^{\prime} \vee z=u \vee z$ by Theorem 3.6(3).

For (1), the case $w \in D_{t}(u)$ for some $u \in U$ is proven above so assume $w \notin D_{t}(u)$ for all $u \in U$. Then $w \notin \bar{D}_{s}(u)$ for all $s \leq t$ and $u \in U$, and since $\Delta_{1}(U)=U \cup D_{A}$ we may assume $t \geq 2$. If $t=2$, then $w \in \Delta_{2}(U)=C_{1}\left(U \cup D_{A}\right)$. If $w \in P_{1}$, we must have $w \in \bar{D}_{1}(u)$ for some $u \in A$ or $w \in B$, both of which are impossible, so $w \notin P_{1}$. Similarly, $w \notin P_{0}$. Since $w \notin \bar{D}_{2}(u)$ for each $u \in U, w \rightarrow B \cup D_{A}$. For $w$ to be in $\Delta_{2}(U)$, we must then have $A \rightarrow w$. Thus, $A \rightarrow w \rightarrow B \cup D_{A}$. Since $|A| \geq 2$ there is a $y \in A$ such that $\bar{D}_{1}(y) \neq \emptyset$ by Theorem 2.1(1). Let $q \in \bar{D}_{1}(y)$. For any $z \in A-\{y\}, z \rightarrow q \rightarrow y$ and $z \rightarrow w \rightarrow q$ so $w \in y \vee z$. Now let $x, z \in U$. If either $x$ or $z$ is in $A$ then $w \in x \vee y \vee z$ as above. If not, then $x, z \in B$ and $y \rightarrow w \rightarrow x$ so $w \in x \vee y \vee z$.

Assume $t>2$ and $w \notin P_{0}$. Since $w \notin \bar{D}_{s}(u)$ for all $s \leq 3$ and $u \in U$, then either $w \rightarrow A \cup D_{B}^{\leftarrow}$ or $A \cup D_{B}^{\leftarrow} \rightarrow w$. Since $w \in \Delta_{t}(U)$ there exist $w^{\prime}, w^{\prime \prime} \in \Delta_{t-1}(U)$ such that $w^{\prime} \rightarrow w \rightarrow w^{\prime \prime}$. Since either $w^{\prime} \rightarrow w \rightarrow A \cup D_{B}^{\leftarrow}$ or $A \cup D_{B}^{\leftarrow} \rightarrow w \rightarrow w^{\prime \prime}$, and since (1) holds for $w^{\prime}$ and $w^{\prime \prime}$ by induction, the result holds for $w$ as well. When $w \notin P_{1}$, a similar argument using $B \cup D_{\vec{A}}$ in place of $A \cup D_{B}^{\leftarrow}$ gives us (1)

For (4), we prove the case $x \in A$, the case $x \in B$ being similar. Let $v \in D_{t}(x)-\{x\}$. Then $v \in \bar{D}_{s}(x)$ for some $s \leq t$. If $s=1$, the result is trivial. For $s \geq 2$, let $s$ be odd, the even case being similar. By the definition of $\bar{D}_{s}(x)$, there exists $v^{\prime} \in \bar{D}_{s-1}(x)$ with $v \rightarrow v^{\prime}$. If $v^{\prime} \neq x$, the result follows by induction. If $v^{\prime}=x$, let $y \in A-\{x\}$. Then Theorem 3.6(2) implies $y \rightarrow v \rightarrow x$, and so $v \in \bar{D}_{1}(x)$, which proves (4).

For (1), we assume for contradiction that $v \in \bar{D}_{t}(x)-\left(P_{0} \cup P_{1}\right)$, where $x \in U$. Thus, $v \neq x$, and so $\bar{D}_{1}(x) \neq \emptyset$ if $x \in A$ and $\bar{D}_{2}(x) \neq \emptyset$ if $x \in B$. We begin with the case $x \in A$. By induction, $t \geq 2$.

Suppose that $t$ is odd. Then there exists $v^{\prime} \in \bar{D}_{t-1}(x)$, with $v \rightarrow v^{\prime}$. We know $v \notin D_{A}$, so either $A \rightarrow v$ or $v \rightarrow A$. Suppose $A \rightarrow v$. Since $|A|,|B| \geq 2$, we can let $y \in A-\{x\}$, 
$z_{1}, z_{2} \in B$. If $v \rightarrow z_{1}$, then $y \rightarrow v \rightarrow z_{1}$ implies $v \in y \vee z_{1} \vee z_{2}$. By induction on $(2), v^{\prime} \rightarrow z_{1}$, and so $v \rightarrow v^{\prime} \rightarrow z_{1}$ implies $v^{\prime} \in y \vee z_{1} \vee z_{2}$. Using induction on (4), $x \in x \vee y \vee z_{1}=v^{\prime} \vee y \vee z_{1} \subseteq y \vee z_{1} \vee z_{2}$, a contradiction. Thus $z_{1} \rightarrow v$. By induction on (4), $v^{\prime} \in x \vee y \vee z_{2}$. Then $x \rightarrow v \rightarrow v^{\prime}$ and $x \rightarrow z_{1} \rightarrow v$, so $z_{1} \in x \vee y \vee z_{2}$, a contradiction.

We now consider the case $v \rightarrow A$. If any vertex in $D_{A} \cup B$ dominates $v$, then $v \in \bar{D}_{2}(y)$ for some $y \in U$ and by induction on (1), $v \in P_{0}$ contrary to our hypothesis. Thus, $v \rightarrow\left(D_{A} \cup B\right)$. Recall that $v \in \Delta_{t}(U)$, and so there exists $w \in \Delta_{t-1}(U)$ with $w \rightarrow v$. Let $z_{1}, z_{2} \in B$. By Lemma 3.7(1), there is a $y \in U$ such that $w \in y \vee z_{1} \vee z_{2}$. Then $w \rightarrow v \rightarrow z_{1}$ implies $v \in y \vee z_{1} \vee z_{2}$ and Lemma 3.5(1) implies $A \subseteq y \vee z_{1} \vee z_{2}$, a contradiction.

If $t$ is even, there is a $v^{\prime} \in \bar{D}_{t-1}(x)$ with $v^{\prime} \rightarrow v$. We begin with the case $v \rightarrow A$. Let $y \in A-\{x\}, z_{1}, z_{2} \in B$. If $z_{1} \rightarrow v$, then $z_{1} \rightarrow v \rightarrow y$ implies $v \in y \vee z_{1}$, and so $x \in y \vee z_{1}$ by Lemma 3.5(1), a contradiction. Thus $v \rightarrow z_{1}$ and the result follows as in the previous paragraph.

In the case $A \rightarrow v$, let $y \in A-\{x\}, z_{1}, z_{2} \in B$. By Theorem 2.1(3) and (5), $v \notin D_{B}^{\leftarrow}$ and we must have either $v \rightarrow B$ or $B \rightarrow v$. In the former case, induction on (2) gives us $y \rightarrow v^{\prime}$. Then $y \rightarrow v \rightarrow z_{1}$ and $y \rightarrow v^{\prime} \rightarrow v$ imply $v, v^{\prime} \in y \vee z_{1} \vee z_{2}$. By induction on (4), we have $x \in x \vee y \vee z_{1}=v^{\prime} \vee y \vee z_{1} \subseteq y \vee z_{1} \vee z_{2}$, a contradiction. If $B \rightarrow v$, we let $w \in \Delta_{t-1}(U)$ with $v \rightarrow w$. By Lemma 3.7(1), there is a $z \in U$ such that $w \in x \vee y \vee z$. Then $x \rightarrow v \rightarrow w$ implies $v \in x \vee y \vee z$, and by Lemma 3.5(2), $B \subseteq x \vee y \vee z$, a contradiction.

For $x \in B$, the result follows from the dual arguments to those in the case $x \in A$, using Lemma 3.5(2) in place of Lemma 3.5(1) and using Lemma 3.5(4) in place of Lemma 3.5(3). This completes the proof of (1).

For (2), we prove the case of $2 k<2 \ell+1$. The other case is similar. Suppose $v \in \bar{D}_{2 k}(x)$, $u \in \bar{D}_{2 \ell+1}(y)$ for some $x, y \in U, x \neq y$ and $u \rightarrow v$. Then there exists $u^{\prime} \in \bar{D}_{2 \ell}(y)$ such that $u \rightarrow u^{\prime}$. Since $u \in \Delta_{2 \ell+1}(U)$ there is a $p \in \Delta_{2 \ell}(U)$ such that $p \rightarrow u$. By Lemma 3.7(1), there is a $z \in U$ such that $p \in z \vee s \vee t$ for any distinct $s, t \in U-\{z\}$.

We first consider the case $z=x$. Let $z_{1}, z_{2} \in U-\{x, y\}$. By Lemma 3.7(1) and induction on (4), $p, v \in x \vee z_{1} \vee z_{2}$. Then $p \rightarrow u \rightarrow v$ implies $u \in x \vee z_{1} \vee z_{2}$. We have $u^{\prime} \rightarrow \cup_{s \neq y} \bar{D}_{1}(s)$ by induction, so if one of $x, z_{1}$, or $z_{2}$ is in $B$, then $u \rightarrow u^{\prime} \rightarrow B-\{y\}$ implies $u^{\prime} \in x \vee z_{1} \vee z_{2}$. Otherwise, Theorem 2.1(1) gives us some $q \in \bar{D}_{1}\left(z_{1}\right) \cup \bar{D}_{1}\left(z_{2}\right)$. Again, $u \rightarrow u^{\prime} \rightarrow q$ gives us $u^{\prime} \in x \vee z_{1} \vee z_{2}$. Induction on (4) gives us $y \in x \vee u^{\prime} \vee z_{1} \subseteq$ $x \vee z_{1} \vee z_{2}$, a contradiction. Identical arguments give us the case $z \notin\{x, y\}$. The case $z=y$ follows similarly, reversing the roles of $x$ and $y$, and reversing the roles of $u^{\prime}$ and $v$. This gives us (2).

For (3), assume $r$ and $s$ are both odd. The even case is similar. Then $u, v \in P_{1}$ by (1). If $u=x$ and $v=y$, the result is obvious. If $u \neq x$ and $v \neq y$, there exists, by definition, $u^{\prime} \in \bar{D}_{r-1}(x)$ and $v^{\prime} \in \bar{D}_{s-1}(y)$ with $u \rightarrow u^{\prime}$ and $v \rightarrow v^{\prime}$. By (2), $u^{\prime} \rightarrow v$ and $v^{\prime} \rightarrow u$. Then clearly $u \vee v=u^{\prime} \vee v^{\prime}$. By induction, $x \vee y=u^{\prime} \vee v^{\prime}$ so $x \vee y=u \vee v$. This leaves, without loss of generality, the case $u=x$ and $v \neq y$. We need only show that $v \in x \vee y$ and $y \in x \vee v$. Since $r$ and $s$ are odd and $x=u$, we must have $x, y \in B$. Since $\bar{D}_{2}(x) \neq \emptyset$, there exists $q \in \bar{D}_{2}(x)$ with $x \rightarrow q$. Since $v \neq y$, there is some $v^{\prime} \in \bar{D}_{s-1}(y)$ with $v \rightarrow v^{\prime}$. By (2), 
$q \rightarrow v$ and $v^{\prime} \rightarrow x$. We then have $q \rightarrow v \rightarrow v^{\prime}$, and so, by induction, $v \in q \vee v^{\prime}=x \vee y$. Similarly, $v \rightarrow v^{\prime} \rightarrow x$, and so $v^{\prime} \in x \vee v$, which implies $y \in x \vee y=q \vee v^{\prime} \subseteq x \vee v$.

For (4), we begin with the case $u \neq x, v \neq y$, and $w \neq z$. If $m, n$, and $p$ have the same parity, say $m, n$, and $p$ are all odd, then there exist $u^{\prime} \in \bar{D}_{m-1}(x), v^{\prime} \in \bar{D}_{n-1}(y)$, and $w^{\prime} \in \bar{D}_{p-1}(x)$ with $u \rightarrow u^{\prime}, v \rightarrow v^{\prime}$, and $w \rightarrow w^{\prime}$. By (2), $\left\{u^{\prime}, v^{\prime}\right\} \rightarrow w,\left\{u^{\prime}, w^{\prime}\right\} \rightarrow v$, and $\left\{v^{\prime}, w^{\prime}\right\} \rightarrow u$. Clearly, $u^{\prime} \vee v^{\prime} \vee w^{\prime}=u \vee v \vee w$. By induction, $x \vee y \vee z=u^{\prime} \vee v^{\prime} \vee w^{\prime}$, giving us the result. If only two of $m, n$, and $p$ have the same parity, say $m$ is odd and $n, p$ are even, we have $u^{\prime}, v^{\prime}$, and $w^{\prime}$ as above with $u \rightarrow u^{\prime}, v^{\prime} \rightarrow v$, and $w^{\prime} \rightarrow w$. By (2), $u^{\prime} \rightarrow\left\{v^{\prime}, w^{\prime}\right\}$, $v \rightarrow\left\{u, w^{\prime}\right\}$, and $w \rightarrow\left\{u, v^{\prime}\right\}$. Again, it is easy to show that $u^{\prime} \vee v^{\prime} \vee w^{\prime}=u \vee v \vee w$, and the result follows as above.

In the case $u=x, v \neq y$, and $w \neq z$, if $n$ and $p$ have the same parity, the result follows similarly as above when $m$ is odd and $n, p$ are even. Suppose $n$ is even and $p$ is odd with $n<p$. Let $w^{\prime} \in \bar{D}_{p-1}(z), v^{\prime} \in \bar{D}_{n-1}$ with $w \rightarrow w^{\prime}$ and $v^{\prime} \rightarrow v$. By induction, $x \vee y \vee z=x \vee v \vee w^{\prime}$. By (2), $w^{\prime} \rightarrow v^{\prime}$ and $v \rightarrow w$. Since $v \rightarrow w \rightarrow w^{\prime}$, it follows that $w \in x \vee v \vee w^{\prime}=x \vee y \vee z$, and so $x \vee v \vee w \subseteq x \vee y \vee z$. For the other direction, suppose $x \in A$. By (2), $x \rightarrow v^{\prime} \rightarrow v$, and so $v^{\prime} \in x \vee v \vee w$. But now $w \rightarrow w^{\prime} \rightarrow v^{\prime}$, and so $w^{\prime} \in x \vee v \vee w$. Thus, $x \vee y \vee z=x \vee v^{\prime} \vee w^{\prime} \subseteq x \vee v \vee w$, which gives us the result. The argument is similar for $x \in B$ and when $p<n$.

The only case remaining is, without loss of generality, $x=u, y=v$, and $z \neq w$. We prove the case $p$ is even, the odd case being similar. Let $w^{\prime} \in \bar{D}_{p-1}(z)$ with $w^{\prime} \rightarrow w$. If $x, y \in A$, then, without loss of generality, we have $q \in \bar{D}_{1}(y)$ with $q \rightarrow y$. By induction, $x \vee y \vee z=x \vee q \vee w^{\prime}$, and we proceed as in the previous paragraph. This leaves us with $x \in A, y \in B$. Let $w^{\prime} \in \bar{D}_{p-1}(z)$ with $w^{\prime} \rightarrow w$. By (2), we have $x \rightarrow\left\{y, w^{\prime}\right\}$ and $w \rightarrow y$. By induction, $x \vee y \vee z=x \vee y \vee w^{\prime}$. Since $w^{\prime} \rightarrow w \rightarrow y$, we have $w \in x \vee y \vee w^{\prime}$, and so $x \vee y \vee w \subseteq x \vee y \vee z$. For the other direction, we have $x \rightarrow w^{\prime} \rightarrow w$, and so $w^{\prime} \in x \vee y \vee w$. We then have $x \vee y \vee z=x \vee y \vee w^{\prime} \subseteq x \vee y \vee w$, and the proof is complete.

This leads to the following corollary.

Corollary 3.8. Let $T$ be a clone-free multipartite tournament and let $U=A \cup B$ be a convexly independent set with $|U| \geq 4$. Then for $x \in U$ the $D(x)$ are pairwise disjoint.

Proof. It suffices to show that the $\bar{D}_{t}(x)$ 's are pairwise disjoint for all $t \geq 0$. Suppose that $v \in \bar{D}_{t}(x) \cap \bar{D}_{t}(y)$, where $x, y \in U$ are distinct. We do the case of $v \in P_{1}$. The case $v \in P_{0}$ is similar. Clearly, we must have $t \geq 2$. Since $v \in \bar{D}_{t}(x)$, there exists $v^{\prime} \in \bar{D}_{t-1}(x)$ with $v \rightarrow v^{\prime}$. But since $v \in \bar{D}_{t}(y)$, Theorem 3.6(2) implies that $v^{\prime} \rightarrow v$, a contradiction.

Before concluding this section we also note that Lemma 3.7 gives the following bound on Caratheodory numbers. This result is also proven in [PWWb] without the hypothesis that $T$ is clone-free.

Corollary 3.9. Let $T$ be a clone-free multipartite tournament. Then the Caratheodory number of $T$ is less than or equal to 3 . 


\section{Helly \& Radon Numbers for Clone-Free Multipar- tite Tournaments}

Let $T$ be a clone-free multipartite tournament and let $U$ be a convexly independent set. As in the previous section let $P_{0}$ and $P_{1}$ be partite sets of $T$ such that $U=A \cup B$ where $A=U \cap P_{0}$ and $B=U \cap P_{1}$. We also assume $|A| \geq|B|, A \rightarrow B$ and $D_{A} \neq \emptyset$ when $B=\emptyset$. We begin by examining $H$ - and $R$-independence for clone-free bipartite tournaments. We require a lemma.

Lemma 4.1. Let $T$ be a clone-free bipartite tournament and let $U$ be a convexly independent set with $|U| \geq 4$.

1. For each $t \geq 0, \Delta_{t}(U)=\bigcup_{x \in U} D_{t}(x)$.

2. $C(U)=\bigcup_{x \in U} D(x)$.

Proof. For (1), note that by definition $\bigcup_{x \in U} D_{t}(x) \subseteq \Delta_{t}(U)$. To show that $\Delta_{t}(U) \subseteq$ $\bigcup_{x \in U} D_{t}(x)$ we induct on $\mathrm{t}$. The case $t=0$ is trivial so assume $v \in \Delta_{t}(U)$ for some $t \geq 1$. If $v \in B$ the result is trivial. Otherwise, there exist $u, w \in \Delta_{t-1}(U)$ such that $u \rightarrow v \rightarrow w$. By induction, $u \in D_{t-1}(x)$ and $w \in D_{t-1}(y)$ for some $x, y \in U$. Then $u \in \bar{D}_{k}(x)$ and $w \in \bar{D}_{l}(y)$ for some $k, l \leq t-1$. Since $T$ is bipartite $u$ and $w$ are in the same partite set so by Theorem 3.6(1), $k$ and $l$ must have the same parity. Then $v \in \bar{D}_{k+1}(x)$ if $k, l$ are odd and $v \in \bar{D}_{l+1}(y)$ if $k, l$ are even. Either way, $v \in \bigcup_{x \in U} D_{t}(x)$ completing the proof of (1). Part (2) follows immediately.

We get the following.

Theorem 4.2. Let $T$ be a clone-free bipartite tournament.

1. Every convexly independent set is $H$-independent.

2. $h(T)=r(T)=d(T)$.

Proof. For (1), let $U=A \cup B$ be a convexly independent set. If $|U| \leq 2$, then clearly $U$ is $H$-independent. In the case $|U| \geq 4$, we have, by Lemma 4.1(2), that $C(U-\{x\}) \subseteq$ $\bigcup_{y \in(U-\{x\})} D(y)$, and so $\bigcap_{x \in U} C(U-\{x\}) \subseteq \bigcap_{x \in U}\left(\bigcup_{y \neq x} D(y)\right)=\emptyset$ since the $D(y)$ 's are pairwise disjoint by Corollary 3.8.

The only remaining case is $|U|=3$. In the case $|A|=2$ and $|B|=1$, let $A=\left\{x_{1}, x_{2}\right\}$ and $B=\{y\}$. We have $C\left(U-\left\{x_{1}\right\}\right)=\left\{x_{2}, y\right\}$ and $C\left(U-\left\{x_{2}\right\}\right)=\left\{x_{1}, y\right\}$. In order for $C\left(U-\left\{x_{1}\right\}\right) \cap C\left(U-\left\{x_{2}\right\}\right) \cap C(U-\{y\}) \neq \emptyset$, we must have $y \in C(U-\{y\})$ which violates the convex independence of $U$. Thus, $U$ is $H$-independent.

Now consider the case $|A|=3, B=\emptyset$. Let $U=\left\{x_{1}, x_{2}, x_{3}\right\}$ be in the partite set $P_{0}$, the other partite set being $P_{1}$. By Theorem 2.1(1), we can assume that there exist $v_{2}, v_{3} \in D_{A}$ with $v_{i} \rightarrow x_{i}$. For contradiction, assume $U$ is $H$-dependent, and let $k$ be minimal such that there exists $v \in\left(x_{1} \vee x_{2}\right) \cap\left(x_{1} \vee x_{3}\right) \cap\left(x_{2} \vee x_{3}\right), v \in C_{k}\left(\left\{x_{1}, x_{2}\right\}\right)$. Clearly, $k \neq 0$. If $k=1$, then $v \in D_{A}$ with either $\left\{x_{1}, x_{3}\right\} \rightarrow v \rightarrow x_{2}$ or $\left\{x_{2}, x_{3}\right\} \rightarrow v \rightarrow x_{1}$. In the 
first case, we have $x_{1} \rightarrow v_{3} \rightarrow x_{3}$ and $v \rightarrow x_{2} \rightarrow v_{3}$, and so $x_{2} \in x_{1} \vee x_{3}$, a contradiction. In the second case, we similarly get $x_{1} \in x_{2} \vee x_{3}$, a contradiction. Thus, $k \geq 2$, and so there exist $w_{1}, w_{2} \in C_{k-1}\left(\left\{x_{1}, x_{2}\right\}\right)$ with $w_{1} \rightarrow v \rightarrow w_{2}$.

If $v \in P_{0}$, then $w_{1}, w_{2} \in P_{1}$. Also note that $v_{2} \in x_{1} \vee x_{2}$. Suppose $w_{1} \rightarrow x_{3}$. Then $w_{1} \rightarrow x_{3} \rightarrow v_{2}$ implies $x_{3} \in x_{1} \vee x_{2}$, a contradiction. Thus, $x_{3} \rightarrow w_{1}$. But then $x_{3} \rightarrow w_{1} \rightarrow v$. Since $v, x_{3} \in\left(x_{1} \vee x_{3}\right) \cap\left(x_{2} \vee x_{3}\right)$, we get $w_{1} \in\left(x_{1} \vee x_{2}\right) \cap\left(x_{1} \vee x_{3}\right) \cap\left(x_{2} \vee x_{3}\right)$. This contradicts the minimality of $k$.

If $v \in P_{1}$, then $w_{1}, w_{2} \in P_{0}$. Suppose $v_{3} \rightarrow w_{2}$. Then $x_{2} \rightarrow v_{3} \rightarrow w_{2}$ and $v_{3} \rightarrow x_{3} \rightarrow v_{2}$ imply that $x_{3} \in x_{1} \vee x_{2}$, a contradiction. Thus, $w_{2} \rightarrow v_{3}$. But then $v \rightarrow w_{2} \rightarrow v_{3}$. Since $v, v_{3} \in\left(x_{1} \vee x_{3}\right) \cap\left(x_{2} \vee x_{3}\right)$, we get $w_{2} \in\left(x_{1} \vee x_{2}\right) \cap\left(x_{1} \vee x_{3}\right) \cap\left(x_{2} \vee x_{3}\right)$. This again contradicts the minimality of $k$, completing the proof of (1). Part (2) follows directly.

In [PWWb], we studied the tripartite tournaments $T_{2 d-1}^{\prime}$, which have partite sets $P_{1}=\left\{x_{1}, \cdots, x_{d-1}\right\}, P_{2}=\left\{y_{1}, y_{2}, \cdots, y_{d-1}\right\}$, and $P_{3}=\{z\}$. The arcs are given by $y_{i} \rightarrow x_{i}$ for all $i \geq 2, x_{i} \rightarrow y_{j}$ otherwise and $P_{1} \rightarrow z \rightarrow P_{2}$. We showed that $h\left(T_{2 d-1}^{\prime}\right)=2$ while $d\left(T_{2 d-1}^{\prime}\right)=d$ for all $d \geq 2$. Furthermore, $r\left(T_{3}^{\prime}\right)=2$ and $r\left(T_{2 d-1}^{\prime}\right)=3$ for all $d \geq 3$. Thus, letting $d \geq 3$ this example shows that we cannot remove the hypothesis that $T$ is bipartite. The following shows that we cannot remove the clone-free hypothesis either.

Proposition 4.3. Let $T$ be the bipartite tournament with vertex set $V=\left\{x_{1}, x_{2}, x_{3}, x_{4}, u\right\}$ and arcs given by $\left\{x_{1}, x_{2}\right\} \rightarrow u \rightarrow\left\{x_{3}, x_{4}\right\}$. Then $d(T)=4$ and $h(T)=r(T)=3$.

Proof. The unique maximum convexly independent set is $S=\left\{x_{1}, x_{2}, x_{3}, x_{4}\right\}$, and so $d(T)=4$. It is easy to see that $u \in \bigcap_{i=1}^{4}\left(S-\left\{x_{i}\right\}\right)$, and so $S$ is $H$-dependent. It is also easy to check that $\left\{x_{1}, x_{2}, x_{3}\right\}$ is $H$-independent, and so $h(T)=3$. Also, $S$ has the Radon partition $\left\{x_{1}, x_{3}\right\} \cup\left\{x_{2}, x_{4}\right\}$, so $S$ is $R$-dependent. Moreover, $\left\{x_{1}, x_{2}, x_{3}\right\}$ is $R$-independent, so $r(T)=3$.

Now we consider clone-free multipartite tournaments. Let $V_{U}=\bigcup_{x \in U} D(x)$. Since $V_{U} \subseteq P_{0} \cup P_{1}$ by Theorem 3.6(1), $V_{U}$ induces a bipartite tournament which we will denote by $T_{U}$.

Lemma 4.4. Let $U=A \cup B$ be a convexly independent set, and let $z \in V-\left(P_{0} \cup P_{1}\right)$.

1. If $|U| \geq 4$ and $z$ distinguishes two vertices in $V_{U}$, then $\left(V_{U} \cap P_{0}\right) \rightarrow z \rightarrow\left(V_{U} \cap P_{1}\right)$.

2. If $|U| \geq 3$ and $z$ distinguishes two vertices in $U \cup D_{\vec{A}}$, then $\left(A \cup D_{B}^{\leftarrow}\right) \rightarrow z \rightarrow\left(B \cup D_{\vec{A}}\right)$

Proof. For (1), Theorem 3.6(1) implies that $z \notin \bar{D}_{t}(x)$ for all $t \geq 0, x \in U$. Thus we cannot have $z \rightarrow u, u \in D_{2 k}(x)$ or $v \rightarrow z, v \in D_{2 k+1}(x)$ for any $k \geq 0, x \in U$. The result follows.

For (2), part (1) proves the result for each case except $|U|=3$. Let $U=\left\{x_{1}, x_{2}, x_{3}\right\}$. If $A=\left\{x_{1}, x_{2}\right\}$ and $B=\left\{x_{3}\right\}$, let $u_{2} \in D_{\vec{A}}$ with $u_{2} \rightarrow x_{2}$. By Theorem 2.1, $z \notin D_{\vec{A}}$, and $u_{2} \in P_{1}$. Thus, without loss of generality, either $A \rightarrow z \rightarrow x_{3}$ or $x_{3} \rightarrow z \rightarrow A$. In the latter case, we have $x_{3} \rightarrow z \rightarrow x_{1}$ and $z \rightarrow x_{2} \rightarrow x_{3}$, and so $x_{2} \in x_{1} \vee x_{3}$, a contradiction. Thus, $A \rightarrow z \rightarrow x_{3}$. Now suppose $u_{2} \rightarrow z$. As before, we get $z \in x_{1} \vee x_{3}$. 
Then $x_{1} \rightarrow u_{2} \rightarrow z$ and $u_{2} \rightarrow x_{2} \rightarrow z$ imply $x_{2} \in x_{1} \vee z$, a contradiction. Showing $D_{B}^{\leftarrow} \rightarrow z$ is similar.

In the case $|A|=3$, let $u_{2}, u_{3} \in D_{\vec{A}}$ with $u_{i} \rightarrow x_{i}$. By Theorem 2.1(3), either $A \rightarrow z \rightarrow D_{A}$ or $D_{A} \rightarrow z \rightarrow A$. In the latter case, since $u_{2} \in x_{1} \vee x_{2}, u_{2} \rightarrow z \rightarrow x_{1}$, and $z \rightarrow x_{3} \rightarrow u_{2}$, we have $x_{3} \in x_{1} \vee x_{2}$, a contradiction. Thus, $A \rightarrow z \rightarrow D_{A}$. Since $D_{B}^{\leftarrow}=\emptyset$, this completes the proof.

Theorem 4.5. Let $T$ be a clone-free multipartite tournament and let $U$ be a convexly independent subset with $|U| \geq 4$, and let $T=(V, E)$ and $T_{U}=\left(V_{U}, E_{U}\right)$ be as above.

1. If $u, v \in V_{U} \cap P_{i}$ with $i \in\{0,1\}, w \in V$ and $u \rightarrow w \rightarrow v$, then $w \in V_{U}$.

2. $T_{U}$ is clone-free.

Proof. For (1), we have $u \in \bar{D}_{k}(x)$ and $v \in \bar{D}_{\ell}(y)$ for some $k, \ell \geq 0$. If $i=0$, then $w \in \bar{D}_{l+1}(y) \subseteq V_{U}$, and if $i=1$, then $w \in \bar{D}_{k+1}(x) \subseteq V_{U}$. Part (2) follows directly.

By Theorem 4.5 and Theorem 4.2, if $U$ is a maximum convexly independent set of $T$, then $h\left(T_{U}\right)=r\left(T_{U}\right)=d\left(T_{U}\right)=|U|$. We now consider the case when $U$ is $H$-independent in $T$.

Theorem 4.6. Let $T$ be a clone-free multipartite tournament and let $U$ be a convexly independent subset of $V$ with $|U| \geq 4$. The following are equivalent.

1. $U$ is $H$-independent.

2. $U$ is $R$-independent.

3. No vertex in $V-\left(P_{0} \cup P_{1}\right)$ distinguishes two vertices in $U \cup D_{\vec{A}}$.

4. No vertex in $V-\left(P_{0} \cup P_{1}\right)$ distinguishes two vertices in $V_{U}$.

5. $C(U)=V_{U}$.

6. There exist three vertices in $U$ that form an $H$-independent set.

Proof. As before, we can assume $U=A \cup B$ with $|A| \geq|B|, A \rightarrow B$ and $D_{\vec{A}} \neq \emptyset$ when $|A| \geq 3$. The implication (1) $\Rightarrow(2)$ is trivial. For (2) $\Rightarrow$ (3), suppose there exists $z \in V-\left(P_{0} \cup P_{1}\right)$ that distinguishes two vertices in $U \cup D_{A}$. By Lemma 4.4(1), $\left(V_{U} \cap P_{0}\right) \rightarrow z \rightarrow\left(V_{U} \cap P_{1}\right)$. If $B \neq \emptyset$, let $x \in A, y \in B, R_{1}=\{x, y\}, R_{2}=U-R_{1}$. Since $x \rightarrow z \rightarrow y, z \in C\left(R_{1}\right)$. Since $|U| \geq 4$ (and thus $|A| \geq 2$ ), then $R_{2} \cap A \neq \emptyset$ and either $R_{2} \cap B \neq \emptyset$ or $C\left(R_{2}\right) \cap D_{A} \neq \emptyset$. In either case, $A \rightarrow z \rightarrow\left(B \cup D_{A}\right)$ implies $z \in C\left(R_{2}\right)$, contradicting $R$-independence. For the case $B=\emptyset$, let $x_{1}, x_{2}, x_{3}, x_{4} \in A$. Without loss of generality, there exist $u_{i} \in D_{A}, i \in\{2,3,4\}$ with $u_{i} \rightarrow x_{i}$ by Theorem 2.1(1). We have $u_{2} \in x_{1} \vee x_{2}$ and $u_{3}, u_{4} \in x_{3} \vee x_{4}$. Since $x_{i} \rightarrow z \rightarrow u_{j}$ for each $i$ and $j$, we get $z \in\left(x_{1} \vee x_{2}\right) \cap\left(x_{3} \vee x_{4}\right)$. Therefore, $\left\{x_{1}, x_{2}\right\}$ and $U-\left\{x_{1}, x_{2}\right\}$ form a Radon partition. 
We now prove $(3) \Rightarrow(4)$. Suppose that $z \in V-\left(P_{0} \cup P_{1}\right)$ distinguishes two vertices in $V_{U}$. Again, Lemma 4.4(1) implies $\left(V_{U} \cap P_{0}\right) \rightarrow z \rightarrow\left(V_{U} \cap P_{1}\right)$. Thus, $z$ distinguishes all vertices in $A$ from all vertices in $B \cup D_{A}$, contrary to (3).

For $(4) \Rightarrow(5)$, it is clear that $V_{U} \subseteq C(U)$. We prove that $C_{n}(U) \subseteq V_{U}$ for all $n \geq 0$ by induction. For $n=0$, the result is obvious. For $n \geq 1$, let $z \in C_{n}(U)$. Then $v \rightarrow z \rightarrow w$ for some $v, w \in C_{n-1}(U)$. By induction, $v, w \in V_{U}$. But since no vertex in $V-\left(P_{0} \cup P_{1}\right)$ can distinguish vertices in $V_{U}$, we must have $z \in P_{0} \cup P_{1}$. Then either $v, w \in P_{0}$ or $v, w \in P_{1}$ so $z \in V_{U}$ by Theorem 4.5.

By Theorem 4.2(1), any convexly independent set in a clone-free bipartite tournament is $H$-independent. Thus, $U$ is $H$-independent in $T_{U}$. Since $C(U)=V_{U}$, this implies that $U$ is $H$-independent in $T$. This gives us both $(5) \Rightarrow(1)$ and $(5) \Rightarrow(6)$

We now prove $(6) \Rightarrow(3)$. Suppose that $z \in V-\left(P_{0} \cup P_{1}\right)$ distinguishes two vertices in $U \cup D_{\vec{A}}$. Lemma 4.4(1) implies $A \cup D_{B}^{\leftarrow} \rightarrow z \rightarrow\left(B \cup D_{\vec{A}}\right)$. Let $u, x, y \in U$. As before, we have $z \in(u \vee x) \cap(u \vee y) \cap(x \vee y)$, and so $\{x, y, z\}$ is $H$-dependent. This proves the result.

As we noted with $T_{2 d-1}^{\prime}$, the Helly number, Radon number, and rank can differ if $d(T) \leq 3$. It is clear that any set of cardinality 1 or 2 is $H$-independent (and thus $R$ - and convexly independent). It is also easy to show that for sets of cardinality 3 , $R$-independence and convex independence are equivalent. Thus, the Helly and Radon number can differ only if $h(T)=2$ and $d(T)=r(T)=3$. More specifically, $R$-independent sets that are $H$-dependent can be characterized as follows.

Theorem 4.7. Let $T$ be a clone-free multipartite tournament and let $U=A \cup B$ be an $R$-independent set, where $|A| \geq|B|, A \rightarrow B$, and $D_{A} \neq 0$ when $|A| \geq 3$. Then $U$ is $H$-dependent if and only if $|U|=3$ and there exists a vertex $z$ in a partite set disjoint from $U \cup D_{A}$ with $A \rightarrow z \rightarrow D_{A}$.

Proof. Suppose $U$ is $H$-dependent. By Theorem 4.6 and the discussion above, $|U|=3$. Let $U=\left\{x_{1}, x_{2}, x_{3}\right\}$. Suppose there does not exist $z \in V(T)$ with $A \rightarrow z \rightarrow D_{A}$. By Lemma 4.4(2), no vertex outside $P_{0} \cup P_{1}$ can distinguish vertices in $U \cup D_{A}$. Thus, if $A=\left\{x_{1}, x_{2}\right\}$ and $B=\left\{x_{3}\right\}$, we have $\left(x_{1} \vee x_{3}\right) \cap\left(x_{2} \vee x_{3}\right)=\left\{x_{3}\right\}$. But $U$ is $H$-dependent, so $x_{3} \in x_{1} \vee x_{2}$, which violates the convex independence of $U$.

Now suppose that $U=A$. By Theorem 2.1(1), without loss of generality there exist $u_{2}, u_{3} \in D_{A}$ with $u_{i} \rightarrow x_{i}$. We claim that $x_{1} \vee x_{2} \subseteq P_{0} \cup P_{1}$. If not, let $z \in\left(x_{1} \vee x_{2}\right)-$ $\left(P_{0} \cup P_{1}\right)$. If $z \rightarrow\left(A \cup D_{A}\right)$, then $z \rightarrow x_{3} \rightarrow u_{2}$ and $z, u_{2} \in x_{1} \vee x_{2}$ imply that $x_{3} \in x_{1} \vee x_{2}$, a contradiction. Otherwise, $\left(A \cup D_{A}\right) \rightarrow z$. In this case, $x_{2} \rightarrow u_{3} \rightarrow z$ and $u_{3} \rightarrow x_{3} \rightarrow x_{2}$ imply that $x_{3} \in x_{1} \vee x_{2}$, a contradiction.

Let $k$ be minimal such that there exists $v \in\left(x_{1} \vee x_{2}\right) \cap\left(x_{1} \vee x_{3}\right) \cap\left(x_{2} \vee x_{3}\right), v \in$ $C_{k}\left(\left\{x_{1}, x_{2}\right\}\right)$. By the above, $v \in P_{0} \cup P_{1}$. We prove the case $v \in P_{1}$, the other case being similar. Clearly, $k \geq 2$, and so there exists $w_{1}, w_{2} \in C_{k-1}\left(\left\{x_{1}, x_{2}\right\}\right)$ such that $w_{1} \rightarrow v \rightarrow w_{2}$. Since each $w_{i} \in\left(x_{1} \vee x_{2}\right)-P_{1}$, we have $w_{i} \in P_{0}$. Suppose $u_{3} \rightarrow w_{2}$. Then $x_{1} \rightarrow u_{3} \rightarrow w_{2}$ and $u_{3} \rightarrow x_{3} \rightarrow u_{2}$ imply that $x_{3} \in x_{1} \vee x_{2}$, a contradiction. Thus, $w_{2} \rightarrow u_{3}$. But then $v \rightarrow w_{2} \rightarrow u_{3}, w_{2} \in x_{1} \vee x_{2}$, and $v, u_{3} \in\left(x_{1} \vee x_{3}\right) \cap\left(x_{2} \vee x_{3}\right)$ imply that $w_{2} \in\left(x_{1} \vee x_{2}\right) \cap\left(x_{1} \vee x_{3}\right) \cap\left(x_{2} \vee x_{3}\right)$, contradicting the minimality of $k$. 
For the converse, if $|U|=3$ and $A \rightarrow z \rightarrow D_{A}$, it is easy to show $z \in \bigcap_{u \in U} C(U-\{u\})$, which makes $U H$-dependent.

The following is immediate from Theorem 4.7. Note that in this result, we assume neither $|A| \geq|B|$ nor $D_{A} \neq \emptyset$.

Corollary 4.8. Let $T$ be a clone-free multipartite tournament. The following are equivalent.

1. $h(T) \neq r(T)$.

2. $h(T)=2$ and $r(T)=3$.

3. For every convexly independent set $U=A \cup B$ of order 3 with $A \neq \emptyset$ and $A \rightarrow B$ when $B \neq \emptyset$, there exists $z \in V(T)$ such that

(a) If $B=\emptyset$ and $D_{A}^{\leftarrow} \neq \emptyset$, then $D_{A}^{\leftarrow} \rightarrow z \rightarrow A$

(b) If $D_{A} \neq \emptyset$, then $A \rightarrow z \rightarrow\left(B \cup D_{\vec{A}}\right)$

As mentioned before, this occurs with the tripartite tournaments $T_{2 d-1}^{\prime}$, where $h\left(T_{2 d-1}^{\prime}\right)=$ 2 and $r\left(T_{2 d-1}^{\prime}\right)=3$ for all $d \geq 3$.

\section{Conclusion}

Our results show that, under two-path convexity, the convex hull of a convexly independent set of vertices contains elements that are particularly well-behaved. They form chains of vertices with alternating edge orientations residing in the same partite sets that contain the convexly independent set and the associated sets $D_{A}$ and $D_{B}^{\leftarrow}$. Furthermore, each of these well-behaved vertices takes on many of the same properties as the vertex that is at the start of its chain. This rich structure enables us to prove that except in some small cases, the Helly number and the Radon number of a clone-free multipartite tournament are the same. Furthermore, the result is stronger in the case of clone-free bipartite tournaments: not only do the Helly number and Radon number coincide, but so does the rank.

The results lead to two obvious questions for further consideration. The class of clonefree multipartite tournaments with Helly number 2 and Radon number 3 seem to have some special properties. It would be nice if we had a way of identifying when such subsets exist, particularly when the Radon number is 3 .

Finally, it is curious to note that while clones may seem innocuous, they clearly impact the structure of the convex subsets in multipartite tournaments. Thus, to what degree can our results be extended to multipartite tournaments with clones? 


\section{References}

[ADEP05] A. Abueida, W.S. Diestelkamp, S.P. Edwards, and D.B. Parker, Determining Properties of a Multipartite Tournament from its Lattice of Convex subsets, Australas. J. Combin. 31 (2005), 217-230.

[CFZ02] G. Chartrand, J.F. Fink, and P. Zhang, Convexity in oriented graphs, Discrete Applied Math. 116 (2002), 115-126.

[CM99] M. Changat and J. Mathew, On triangle path convexity in graphs, Discrete Math. 206 (1999), 91-95.

[CZ99] G. Chartrand and P. Zhang, Convex sets in graphs, Cong. Numer. 136 (1999), 19-32.

[Duc88] P. Duchet, Convexity in graphs II. Minimal path convexity, J. Combin. Theory, Ser. B 44 (1988), 307-316.

[EFHM72] P. Erdös, E. Fried, A. Hajnal, and E.C. Milner, Some remarks on simple tournaments, Algebra Universalis 2 (1972), 238-245.

[ES85] M.G. Everett and S.B. Seidman, The hull number of a graph, Discrete Math. 57 (1985), 217-223.

[HN81] F. Harary and J. Nieminen, Convexity in graphs, J. Differential Geometry 16 (1981), 185-190.

[HW96] D.J. Haglin and M.J. Wolf, On convex subsets in tournaments, SIAM Journal on Discrete Mathematics 9 (1996), 63-70.

[JN84] R.E. Jamison and R. Nowakowski, A Helly theorem for convexity in graphs, Discrete Math. 51 (1984), 35-39.

[Moo72] J.W. Moon, Embedding tournaments in simple tournaments, Discrete Math. 2 (1972), 389-395.

[Nie81] J. Nieminen, On path- and geodesic-convexity in digraphs, Glasnik Matematicki 16 (1981), 193-197.

[Pfa71] J.L. Pfaltz, Convexity in directed graphs, J. Combinatorial Theory 10 (1971), $143-152$.

[Pol95] N. Polat, A Helly theorem for geodesic convexity in strongly dismantlable graphs, Discrete Math. 140 (1995), 119-127.

[PWWa] D.B. Parker, R.F. Westhoff, and M.J. Wolf, Convex independence and the structure of clone-free multipartite tournaments, Submitted. 
[PWWb] _ On two-path convexity in multipartite tournaments, Submitted.

[PWWc] _ Two-path convexity and bipartite tournaments of small rank, To appear in Ars Combin.

[PWWd] _ Two-path convexity in clone-free regular multipartite tournaments, To appear in Australas. J. Combin.

[Var76] J.C. Varlet, Convexity in Tournaments, Bull. Societe Royale des Sciences de Liege 45 (1976), 570-586.

[vdV93] M.L.J van de Vel, Theory of Convex Structures, North Holland, Amsterdam, 1993. 